\title{
The Study of Subway Tunnel Seismic Nonlinear Dynamic Response
}

\author{
Mingming Liu 1 \\ Xijing University, Xi'an, 710123, China \\ e-mail: 123276393@qq.com \\ Shidan He3 \\ Xijing University, Xi'an, 710123, China \\ e-mail:315705804@qq.com
}

\author{
Xiaofang Li2 \\ Xijing University, Xi'an, 710123, China \\ e-mail: 563237924@qq.com
}

\begin{abstract}
Objective: This paper studies the subway tunnel seismic nonlinear dynamic response, so that can get some helpful conclusions, which is helpful for seismic designing of metro tunnel. Methods:A series of probems on dynamic FEM are discussed and suggestions about setting of soil dynamic constitutive model and soil-structure contact model are given in the paper.With the example of Tianjin No.1 subway and No.2 sub-way, nonlinear analysis of dynamic response including displacement,stress and strain of circular tunnel and displacement of the surface of ground are conducted to draw some helpful conclusions. Results: Application of dynamic finite element method for seismic response of metro tunnel to do nonlinear analysis, can get a series of laws and conclusions. These laws apply to circular shield tunnel. Conclusion: Application of dynamic finite element method for seismic response of metro tunnel to do nonlinear analysis, draw some useful rules and conclusions, which will provide reference and basis for seismic design and research of the subway tunnel.
\end{abstract}

Keywords-Component; subway tunnel; Seismic; nonlinear dynamic response

\section{INTRODUCTION}

With the development of modern society and economy, the city expanding, urban population soaring, urban traffic problems is increasingly becoming a "bottleneck" for the urban development. Ground transportation tension also prompted people to start developing this important resource of underground space.Underground traffic which is the core of subway with its comfortable and safe, efficient and convenient, energy saving and environmental protection and other significant advantages of urban transport is becoming an important part.

Subway is an important lifeline engineering. Because of a lot of cities are located in high-intensity fortified area, therefore the seismic analysis and research of subway structure has important theoretical and engineering significance. The traditional method for tunnel seismic analysis are quasi-static method. Although they are easy to calculate, but due to many assumptions, can not accurately consider the impact of nonlinear, heterogeneity and complexity of the boundary changes and other factors, so it is difficult to consider the dynamic between soil and structure interaction.With the development of computers and computing theories, dynamic finite element method as a representative of numerical methods came into being. It avoids the shortcomings of the above theory, provides a powerful weapon for the in-depth study of the seismic characteristics of underground structures under complex situations.

Based on this, this paper studies the subway tunnel seismic nonlinear dynamic response. This paper mainly do that A series of probems on dynamic FEM are discussed and suggestions about setting of soil dynamic constitutive model and soil-structure contact model are given in the paper.With the example of Tianjin No.1 subway and No.2 sub-way, nonlinear analysis of dynamic response including displacement,stress and strain of circular tunnel and displacement of the surface of ground are conducted to draw some helpful conclusions, which will provide reference and basis for seismic design and research of the subway tunnel.

\section{II. THE DYNAMIC FINITE ELEMENT METHOD OF}

NONLINEAR ANALYSIS OF SEISMIC RESPONSE OF METRO TUNNEL

\section{A. Soil nonlinear materialsand soil dynamic constitutive model}

Soil and tunnel structure interaction under seismic loads, the power will be reflected in an obvious response linearity, hysteresis and deformation cumulative. In order to take full account of these dynamic properties of soil in cyclic loading and lower body exhibits non-linear characteristics, we use multiple linear kinematic hardening model as soil dynamic constitutive model.

Multilinear kinematic hardening model usesBesseling model (also known as sub-surface model or level model) to describe dynamic characteristics of the elastic-plastic material. It is assumed that the material constituted by a plurality of sub-surface. All sub-surface to produce the same strain, but each has a different yield strength. The total stress-strain relationship, but they can be combined to describe the various complex nature of the material, forming - - although each sub surface simply use ideal plastic stress-strain curve can be used to represent multisegment polyline. Each break point on the curve represents the yield performance of a sub-surface.

In the application of this model, just given elastic modulus, Poisson's ratio and the stress-strain curve break point coordinates, you can determine the stress-strain curve, and then incorporated into the dynamic equilibrium equations of nonlinear solution process. 


\section{B. Contact nonlinearity and nonlinear simulation between soil and structure}

In the dynamic analysis systems, nonlinear interactions achieve between soil and tunnel structure by setting appropriate contact surface. In order to fully reflect the relative slip, detachment, and then closed between soil and tunnel structure between the contact surfaces, we use surface - surface contact friction elements to simulate changes in the status of the contact surface of the complex as a contact surface model.

Face - face contact model general steps are as follows:

According to the specific circumstances of the contact problem, the contact surface on both sides of the boundary surface materials are designated as "target surface" and "contact surfaces";

In the finite element mesh, which has discrete two materials, do set respectively on the boundary surfaces, which matches the common unit interface element;

Select and define the various model parameters;

The method of determining solving;

Incorporated into the finite element analysis, finite element - contact element coupled model is solved.

In the above step, the model parameters of the model selection and determination are key applications.

C. Determine damping

The project will generally be divided into external damping damping and internal damping two categories. Outside sources including sliding friction damping between two solid surfaces, and study of the system and its external liquid, gas and other energy loss caused by the interaction of the like; damping materials is through internal friction energy is converted to heat dissipation.

In practical dynamic analysis and practical engineering, the most widely used is the Rayleigh damping.

$$
C=\alpha M+\beta K
$$

Among:

$\mathrm{C}$ refers to overall damping matrix; $\mathrm{M}$ refers to overall quality matrix; $\mathrm{K}$ refers to overall stiffness matrix.

$$
\begin{aligned}
\alpha=\frac{2 \omega_{i} \omega_{j}\left(\omega_{i} \xi_{j}-\omega_{j} \xi_{i}\right)}{\omega_{i}^{2}-\omega_{j}^{2}} \\
\beta=\frac{2\left(\omega_{i} \xi_{j}-\omega_{j} \xi_{i}\right)}{\omega_{i}^{2}-\omega_{j}^{2}}
\end{aligned}
$$

Among:

$\omega_{i}$ and $\omega_{j}$ refer to the $\mathrm{i}$ and $\mathrm{j}$-th natural circular frequency; $\xi_{i}$ and $\xi_{j}$ refer to the $\mathrm{i}$ and $\mathrm{j}$-th modal damping ratio.

\section{Select the boundary conditions}

Boundary conditions are generally calculated according to the nature of its input model system, as well as analytical methods to determine the dynamic load. Because this material nonlinear constitutive model and the contact surface models, but also to solve the system in the time domain, so this boundary condition is assumed as follows:
Bottom boundary (ie bedrock Office) assumed the horizontal and vertical directions are fixed;

Lateral boundaries. Due to the current seismic design in seismic shear waves inputs are mostly horizontal vibration, away from the structure of the formation of the vertical deformation can be ignored, so the lateral displacement of the horizontal boundary to take free, only the vertical restraint direction of displacement; Top border. Horizontal and vertical directions are free.

E. Select cell division and computational domain

Under normal circumstances, the unit mesh finer, higher precision, but the greater the amount of computation required to calculate longer time. Therefore, a reasonable choice of unit size and mesh shape is very important. Based on the research needs of the stress and strain complex and important lining and lining shape according to the surrounding soil characteristics, using a uniform dense grid divided by lining the sides level and the depth direction with increasing distance from the grid can be gradually zoom. This will not only meet the research needs, and reasonable to reduce the amount of calculation and computation time.

Meshing range is an important issue unit meshing. If the selected range is too large, although the results more accurate, but the computation time is too long and the high demand for the machine's memory. If the partition range is too small, the results will have a greater impact of human error by the border. Real calculation results show that when taken as scoping 8D 10D (D for the tunnel width or height) or more, you can get a better accuracy.

\section{CASE ANALYSIS}

\section{A. Case analysis and finite element modeling}

Geological and engineering data related to this case from the Tianjin Metro Line 1 and Line 2 under construction projects. Line 1 and Line 2 new sections are used in most sections shield construction. Sectional shape of a circular tunnel, the tunnel using precast concrete segments assembled from the annular lining structure. The area seismic intensity of 7 degrees. Most areas III class venue.

Calculation of soil ---- tunnel system diagram is shown as Figure 1. Among, The thickness of the concrete lining of the ring is $0.35 \mathrm{~m}$, its outer diameter of $6.2 \mathrm{~m}$, an inner diameter of $5.5 \mathrm{~m}$, top tunnel Jude Biao $10 \mathrm{~m}$. According meshing range exposition, soil - FEM-area tunnel system is taken as $50 \mathrm{~m} \times 40 \mathrm{~m}$ shown in Fig .2.Calculation of soil and soil within the region are used in lining flat four-node isoparametric elements to divide. Set between the soil surface and lining - surface contact friction unit.

In this paper, the Tianjin Ninghe wave of north-south seismic records. The peak acceleration of ground motion as $145.80 \mathrm{Gal}(1 \mathrm{Gal}=1 \mathrm{~cm} / \mathrm{s} 2=0.01 \mathrm{~m} / \mathrm{s} 2)$, the sampling frequency is $0.01 \mathrm{~s}$, duration of $19.19 \mathrm{~s}$. This article from the bedrock at the input horizontal direction seismic waves. To save computing time, the interception of the acceleration curve $6 s \sim 16 \mathrm{~s}$ period. According to the provisions of GB50011- 2001 "Seismic Design of Buildings", in this case basis having VII degrees fortification, III class venue operators, rare earthquake standards occurs, maximum acceleration adjusted 310Gal $(3.10 \mathrm{~m} / \mathrm{s} 2)$, as shown in Fig.. 
In Figure 3, the horizontal axis represents time and the vertical axis represents acceleration

\section{B. Process curve analysis of system dynamic response}

Take lining top node 575, node 591 and the bottom edge of the outer sides of the nodes 574 and 583 to study, which is shown as Fig .4 Give each node dynamic time history response curve by calculation. After analysis, obtain the following rules:

Horizontal displacement of the peak of each point is much larger than its peak vertical displacement, the difference of more than 5 times. Most of the points in time when the earthquake process, its horizontal displacement is greater than its vertical displacement.

According to the curve dynamic of dynamic principal stress and principal strain of each node, vibration system can be estimated period of about $2 \mathrm{~s}$. The vibration period and site conditions and seismic wave input related.

C. The maximum dynamic response and dynamic response Dynamic contour analysis

Lining hoop stress maximum is $1560 \mathrm{kPa}$, and the absolute value of the maximum radial stress only $35.5 \mathrm{kPa}$, a difference of two orders of magnitude. In addition, the inner lining, the outer edge of the absolute value of the stress is similar and opposite sign. Thus, at this moment mainly in LINING.

You can get the dynamic contours of lining stress field of the whole process by calculation. Using hoop stress to represent its stress state), you can get power circular hole tunnel under horizontal seismic loads response law:

After the vibration into a stable state, most of the time, the stress concentration area are located in the lining Xtype oblique diagonal area; The maximum dynamic stress often appear in the lining of the inside of the location, and in the upper part of the majority.

Thus it can be used for the following analysis: When the lining thickness when the relative small size Diameter (most projects lining for such situations), its main internal forces moment. Therefore its force deformation mainly concentrated in certain orthogonal diagonal portions, the lining of a corresponding position two diagonal inwardly (ie the inside of tension and lateral compression), the other two diagonal to the corresponding position the outer curved (ie outside of tension, the inner compression). For the lining of the dynamic response under horizontal seismic loads, stress concentration areas are located in "X" type diagonal oblique Location: In contrast, under static conditions such as gravity stress concentration area focused on lining "cross" type diagonal position.

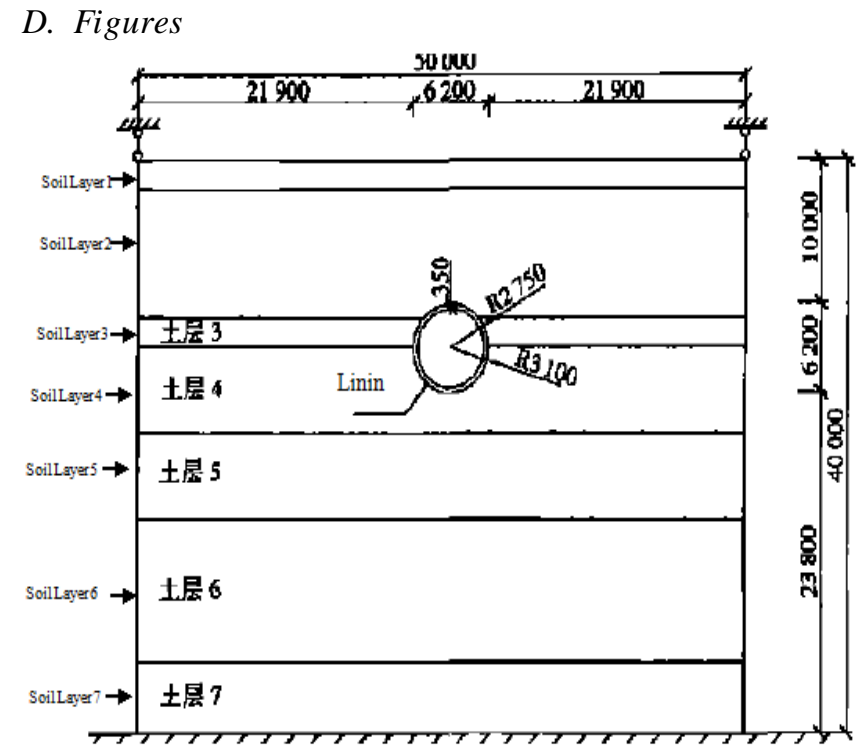

Figure 1. Soil - tunnel system's calculation diagram

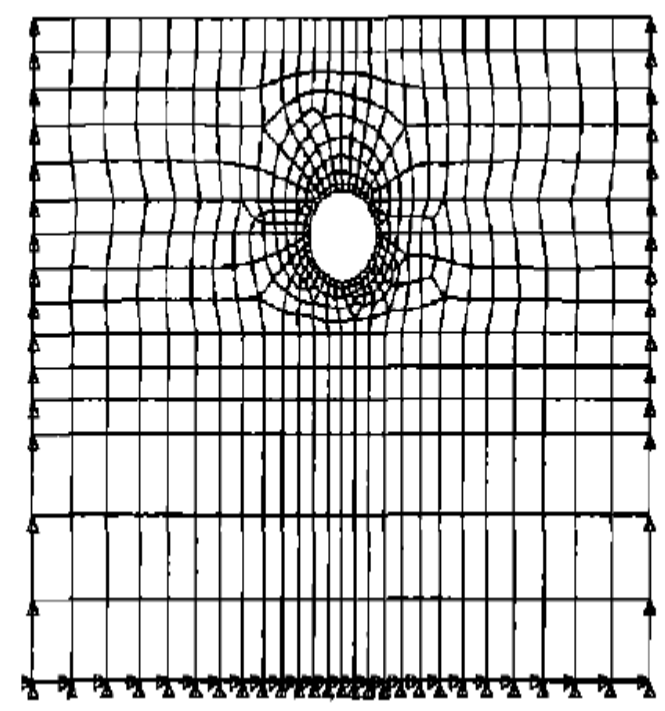

Figure 2. Soil - tunnel system's finite element model

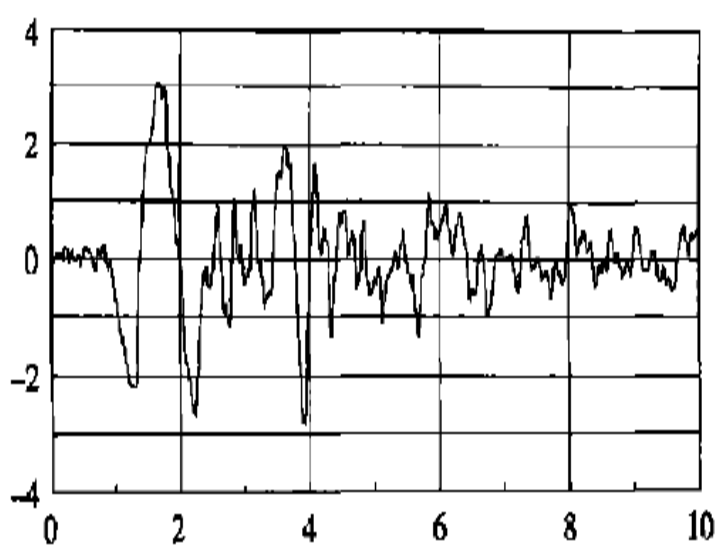

Figure 3. Ninghe Tianjin Bonan northbound acceleration time curve 


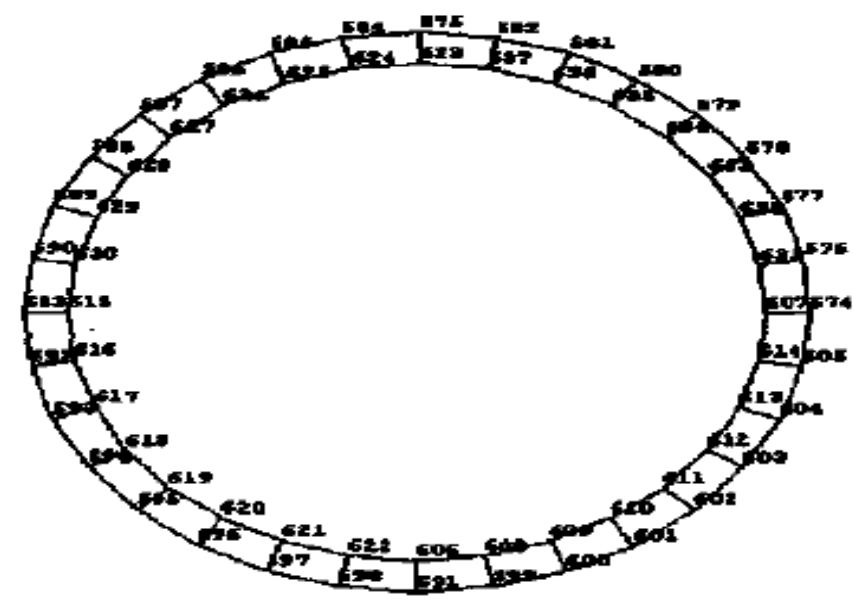

Figure 4. Lining node number

IV.

\section{CONCLUSIONS}

This paper mainly do that A series of probems on dynamic FEM are discussed and suggestions about setting of soil dynamic constitutive model and soil-structure contact model are given in the paper.With the example of Tianjin No.1 subway and No.2 sub-way, nonlinear analysis of dynamic response including displacement,stress and strain of circular tunnel and displacement of the surface of ground are conducted to draw some helpful conclusions, which will provide reference and basis for seismic design and research of the subway tunnel.

Conclusions are as follows:

1 most cases, the lining under horizontal seismic horizontal displacement of each point is much larger than its vertical displacement;

2 When lining thickness is moderate, its main internal forces moment. In the horizontal seismic loads, lining stress concentration areas are located in " $\mathrm{X}$ " type diagonal oblique position. One of the two diagonal positions corresponding to bent inwardly (i.e., inner tension, lateral pressure), the other two outwardly bent position corresponding to the diagonal (i.e., the outer tension, the inside pressure). Multi-peak dynamic stress caused by the circular inner lining bend, and greater stress generated in the upper curved area. Deal with the above characteristics circular shield tunnel in seismic design given attention. Be strengthened for the corresponding parts by calculation or construction measures.

3 The horizontal displacement of surface points is relatively large, which is unfavorable seismic ground structure. The surface is much smaller than the vertical displacement of the points of its horizontal displacement. Both surface seismic subsidence also uplift, surface seismic subsidence small bulge relatively large number, but all belong to the safe range.

\section{REFERENCES}

[1] Besseling J. F.. "A theory of elastic, plastic, and creep deformationsof an initially isotropic material showing anisotropic strain-hardening, creep recovery, and secondary creep" J. Journal of Applied Mechanics, 1958, pp. 529 536.

[2] Owen R. J. Prakash A. Zienkiewicz O. C."Finite Element Analysis of Non-Linear Composite Materials by Use of Overlay Systems". Computers and Structures, 1974, 4, pp. 1251 1267.

[3] H. Zhang. "Based on the soil - structure interaction theory of nonlinear dynamic analysis of Subway tunnel earthquake" J. Tianjin: Tianjin University, 2004. pp.29 32.

[4] Wang Gang; Sun Bo. "Study on Seismic Response of Shield Tunnel Under Complex Geological Conditions"J. Journal of Shandong Agricultural University. Natural Science, 2014,45,pp. 69-76.

[5] Li Zhongxian; Wang Honglong; Li Ning. "Seismic response analysis of shield tunnel consideringbmechanical characteristics of joints" J. Earthquake Engineering and Engineering Vibration., 2012, 22, pp.166-173.

[6] Ye Daokui; Chen Guoxing; Zuo Xi. "Comparative Analysis between Numerical Simulation and Model Test on the Seismic Response of Subway Station Structure in Soft Clay" J. Journal of Disaster Prevention and Mitigation Engineering, 2012, 32, pp. 5.

[7] Zhuang, HY; Chen, GX; Xu, Y. "Study on the influence of shallow excavated tunnels in soft site on design ground motion parameters". 3rd International Conference on Earthquake Engineering, 2004, pp. 91-97.

[8] K. H. Xie. J. Zhou. "Geotechnical engineering finite element analysis theory and applications", Beijing, Science Press, 2002, pp. 25-41.

[9] Zhang Hong; Bi Jihong; Zhang Wei. "Nonlinear analysis of seismic response of metro tunnel" J. Earthquake Engineering and Engineering Vibration. 2004, 24, pp. 146-153.

[10] Dafalias Y. F. and Popov E. P. A model of nonlinearly hardening materials for complex loadings. Acta. Mechanics. 1975, 21, pp. $173 \sim 192$. 